\title{
Calcificación de aorta abdominal de pacientes en hemodiálisis en una unidad renal
}

\section{Calcification of the abdominal aorta in patients on hemodialysis in a renal unit}

\author{
Luis A. Valderrama, Luis Fernando Guerrero, Guillermo Bolívar • \\ Cali (Colombia)
}

\section{Resumen}

Objetivo: describir la prevalencia de calcificaciones de aorta abdominal y factores de riesgo relacionados de pacientes en hemodiálisis en una unidad renal de la ciudad de Cali.

Introducción: las calcificaciones vasculares son prevalentes en pacientes con enfermedad renal crónica (ERC). Los pacientes en hemodiálisis presentan mayor riesgo de mortalidad cardiovascular y factores como la edad, enfermedades asociadas y algunas alteraciones metabólicas incrementan el riesgo de calcificaciones vasculares.

Material y métodos: se incluyeron 47 pacientes en hemodiálisis mayores de 18 años y con más de tres meses en hemodiálisis. La radiografía lumbar lateral fue usada para evaluar las calcificaciones de los segmentos L1 a L4 utilizando un puntaje para su cuantificación y un único observador entrenado. Se realizaron mediciones de calcio, fósforo, PTH, colesterol, triglicéridos en sangre y tensión arterial. Fue utilizado análisis de regresión para determinar asociaciones entre las calcificaciones de aorta abdominal y las características de los pacientes.

Resultados: se encontró que la edad promedio fue de 60,8 años (rango 25-84), el 57.4\% fueron mujeres, el tiempo promedio en hemodiálisis 44.6 meses (rango 3-156). El 63.8\% de los pacientes presentaron calcificaciones en algún segmento de la aorta abdominal. Se encontró que a mayor edad $(\mathrm{P}=0.0006)$ y menor tensión arterial diastólica $(\mathrm{TAD})(\mathrm{P}=0.036)$, el riesgo de prevalencia de calcificación es mayor.

Conclusiones: la calcificación de aorta abdominal es prevalente en los pacientes con ERC en hemodiálisis. Factores como la edad y la disminución de la TAD se encontraron asociadas al grupo de calcificaciones. La radiografía lumbar lateral es una herramienta ampliamente disponible y menos costosa que puede ser tenida en cuenta en el tratamiento y evaluación de riesgo cardiovascular en los pacientes en hemodiálisis y como predictor de complicaciones. (Acta Med Colom 2012; 37: 14-20).

Palabras clave: enfermedad renal crónica, hemodiálisis, calcificación, radiografía lumbar lateral.

\section{Abstract}

Objective: to describe the prevalence of abdominal aortic calcifications and risk factors in patients on hemodialysis in the renal unit Imbanaco Medical Center in the city of Cali.

Background: vascular calcification is prevalent in patients with chronic kidney disease. Hemodialysis (HD) patients are at increased risk of cardiovascular mortality and factors such as age, associated diseases and some metabolic abnormalities increase the risk of vascular calcification.

Material and methods: we included 47 hemodialysis patients over 18 years and more than three months in dialysis. The lateral lumbar radiography was used to assess the calcification of the segments L1 to L4 using a score for quantification and a single trained observer. Regression analysis was used to determine associations between AAC and patient characteristics.

Results: median age of patients was 60.8 years (range $25-84$ ), $57.4 \%$ were female, median duration of HD 44.6 months (range 3-156). We found that in $63.8 \%$ of patients had calcifications in any segment of the abdominal aorta. We found that older age $(\mathrm{P}=0.0006)$ and lower diastolic blood pressure
Dr. Luis A. Valderrama: Internista, Nefrólogo, Director Médico Unidad Renal Centro Médico Imbanaco, Corporación Comfenalco Universidad Libre, Docente de la Universidad Libre; Dr. Luis Fernando Guerrero G.: Residente de Tercer año de Medicina Interna, Universidad Libre; Dr. Guillermo Bolívar: Radiólogo, Clínica de Occidente, Docente de la Universidad Libre, Cali. Cali (Colombia).

Institución donde se realizó el trabajo: Unidad Renal Fresenius Medical Care - Centro médico Imbanaco. Cali (Colombia).

Patrocinio: Recursos Propios.

Conflicto de intereses: Ninguno Correspondencia. Dr. Luis A. Valderrama. Cali (Colombia)

E-mail: luvaldeco@hotmail.com Recibido: 11/V/2011 Aceptado: 26/I/2012 
(DBP) $(\mathrm{P}=0.036)$ the risk is greater prevalence of calcification.

Conclusions: abdominal aortic calcification is prevalent in patients with CKD on hemodialysis. Factors such as age and decreased DBP were associated with the group of calcifications.The lateral lumbar radiograph is a tool widely available and less expensive than can be taken into account in the assessment of cardiovascular risk in hemodialysis patients and as a predictor of complications. (Acta Med Colom 2012; 37: 14-20).

Key words: chronic kidney disease, hemodyalisis, calcification, radiography lumbosacral region.

\section{Introducción}

La enfermedad renal crónica (ERC) es una patología catastrófica en la cual se presenta una pérdida progresiva e irreversible de la función renal. Dicha condición clínica constituye un importante problema de salud pública, en el ámbito mundial y nacional (1).

Pacientes con ERC en diálisis tienen un incremento en la ateroesclerosis, la cual frecuentemente progresa durante cortos periodos. Este fenómeno afecta aún a pacientes jóvenes en diálisis, y probablemente explique en parte, por qué la mortalidad cardiovascular se incrementa 20 a 30 veces en este grupo (2). Varios estudios han demostrado que los puntajes de calcificación arterial y de inflamación crónica predicen mortalidad cardiovascular y están asociados estrechamente a los pacientes en hemodiálisis $(3,4)$. La calcificación de aorta abdominal también ha sido asociada a incremento en el riesgo de falla cardiaca en el estudio de Framingham (15).

La sobrecarga de calcio podría estar relacionada con las calcificaciones arteriales $(5,6)$. Igualmente las concentraciones de calcio utilizadas en hemodiálisis pueden empeorar el progreso de las calcificaciones vasculares, lo cual se ha evidenciado en algunos estudios (7).

La evaluación de las calcificaciones vasculares puede ser realizada a través de tomografía computarizada helicoidal; sin embargo, este método requiere equipos especiales, es costoso y no es fácilmente disponible en la práctica clínica diaria $(16,17)$.

La radiografía lumbar lateral es un método sencillo, menos costoso que ha sido utilizado en varios estudios para encontrar calcificaciones en pacientes en hemodiálisis $(8-10,18)$.

En Colombia no tenemos datos publicados de la prevalencia de calcificaciones de aorta abdominal (CAA), que se presentan en los pacientes en hemodiálisis, por lo cual el estudio pretende describir la prevalencia de dichas calcificaciones y factores de riesgo relacionados con su aparición en pacientes de una unidad renal.

\section{Material y métodos}

La población estuvo conformada por 162 pacientes en terapia de reemplazo renal tipo hemodiálisis de la unidad renal Fresenius Medical Care del Centro Médico de Imbanaco en la ciudad de Cali. La muestra poblacional fue seleccionada mediante un muestreo aleatorio simple, con un nivel de confianza de $95 \%$ y un error de $5 \%$, mediante el programa estadístico Epi Info. Se seleccionó un total de 52 pacientes, ajustado a una tasa de no respuesta de $20 \%$.

Los criterios de inclusión de los pacientes fueron la edad (mayor de 18 años), tiempo de hemodiálisis mayor de tresmeses y aceptación de ingreso al estudio con consentimiento informado. Los criterios de exclusión se determinaron por la imposibilidad de medir los parámetros de rigidez arterial (ausencia de pulsos, fibrilación auricular, fístulas arteriovenosas bilaterales). Al final, cinco pacientes no se realizaron la radiografía lumbar, por lo cual se incluyeron 47 pacientes.

Se recolectaron los datos de información demográfica, tiempo en hemodiálisis, antecedentes de comorbilidades (diabetes mellitus, hipertensión arterial, enfermedad coronaria, accidente cerebrovascular, tabaquismo), tensión arterial, medicamentos formulados y se obtuvieron resultados de laboratorios de calcio, paratohormona (PTH), fósforo, colesterol total y triglicéridos en sangre.

Se realizó una radiografía lumbar lateral a cada paciente utilizando un mismo equipo (GE-Proteus XR/a) y las radiografías fueron leídas por un mismo radiólogo entrenado, utilizando un formato para evaluación del puntaje de calcificación (Tabla 1, Figura 1).

Se calculó el puntaje de CAA con el sistema utilizado en el estudio CORD (Calcification Outcome in Renal Disease) previamente validado en otros estudios (8-10). Los segmentos evaluados de la radiografía fueron L1 a L4.

\section{Análisis estadístico}

Los datos fueron procesados mediante el programa estadístico SPSS versión 18.0. Se realizó un análisis descriptivo,

Tabla 1. Puntaje de calcificación de aorta abdominal.

\begin{tabular}{|c|c|c|c|c|}
\hline Nivel & $\begin{array}{c}\text { Segmento } \\
\text { afectado }\end{array}$ & $\begin{array}{c}\text { Pared } \\
\text { posterior } \\
(\mathbf{0 - 3})\end{array}$ & $\begin{array}{c}\text { Pared } \\
\text { anterior } \\
\mathbf{( 0 - 3 )}\end{array}$ & $\begin{array}{c}\text { Puntaje } \\
\text { compuesto (CAA) } \\
(\mathbf{0 - 6})\end{array}$ \\
\hline L1 & & & & \\
L2 & & & & \\
L4 & & 12 & 12 & 24 \\
Total & & & & \\
\hline Máximo & & & & \\
\hline \\
Grados: $0=$ No calcificaciones. $1=$ Pequeños depósitos de calcificación que llenan \\
menos de $1 / 3$ de la longitud de la pared de la aorta. $2=1 / 3$ a $2 / 3$ de la pared calcificada. \\
3=2/3 o más de la pared calcificada \\
\hline
\end{tabular}





Figura 1. Radiografía lateral lumbar de dos pacientes. La figura A, muestra depósitos de calcio en toda la aorta abdominal (puntaje compuesto de CAA de 24) y la figura B muestra algunos depósitos de calcio (puntaje compuesto de CAA de 11).

el cual contiene tablas de frecuencias para variables cualitativas y estadísticos descriptivos como media y desviación estándar para las variables cuantitativas. También se aplicaron las pruebas de significancia de las variables cuantitativas por sexo, mediante la prueba t-student y el cálculo de Odds ratio y prueba $C h i^{2}$ para las variables cualitativas. Igualmente se realizó un análisis de regresión logística binominal para determinar asociaciones significativas entre calcificación de aorta abdominal y otras variables.

\section{Resultados}

De los 47 pacientes en hemodiálisis, el 57.4\% $(n=27)$ correspondieron al sexo femenino, con edades comprendidas entre los 25 y 84 años, el tiempo promedio de hemodiálisis de la población fue de 44.6 meses. La Tabla 2 muestra las variables edad (años), tiempo en hemodiálisis (meses), presión arterial sistólica (TAS) y presión arterial diastólica (TAD), distribuidas según el sexo. No se encontraron diferencias significativas de las variables edad, TAS, TAD y tiempo en hemodiálisis, por sexo. Sin embargo, se encontró que a mayor edad y menor TAD aumenta la probabilidad de riesgo de tener calcificaciones en la aorta abdominal (Figuras 2 y 3 ).

Al analizar los antecedentes de los pacientes, se encontró que $38 \%$ (n:18) de la población tenían antecedentes de diabe-

Tabla 2. Estadísticos descriptivos de la edad, tiempo en hemodiálisis y presión arterial, según sexo.

\begin{tabular}{|c|c|c|c|c|c|c|c|}
\hline Variables & Sexo & $\mathbf{N}$ & Mínimo & Máximo & Promedio & $\pm \mathrm{DS}$ & $\mathbf{P}$ \\
\hline Edad (años) & $\begin{array}{c}\mathrm{F} \\
\mathrm{M} \\
\text { Total }\end{array}$ & $\begin{array}{l}27 \\
20 \\
47\end{array}$ & $\begin{array}{l}25 \\
35 \\
25\end{array}$ & $\begin{array}{l}84 \\
77 \\
84\end{array}$ & $\begin{array}{l}60.5 \\
61.2 \\
60.8\end{array}$ & $\begin{array}{l}16.1 \\
11.7 \\
14.2\end{array}$ & 0.8664 \\
\hline Tiempo en hemodiálisis (meses) & $\begin{array}{c}\mathrm{F} \\
\mathrm{M} \\
\text { Total }\end{array}$ & $\begin{array}{l}27 \\
20 \\
47\end{array}$ & $\begin{array}{l}3 \\
3 \\
3\end{array}$ & $\begin{array}{l}156 \\
134 \\
156\end{array}$ & $\begin{array}{l}39.7 \\
51.2 \\
44.6\end{array}$ & $\begin{array}{l}40.1 \\
32.8 \\
37.2\end{array}$ & 0.3025 \\
\hline TAS (mmHg) & $\begin{array}{c}\mathrm{F} \\
\mathrm{M} \\
\text { Total }\end{array}$ & $\begin{array}{l}27 \\
20 \\
47\end{array}$ & $\begin{array}{l}111 \\
114 \\
111\end{array}$ & $\begin{array}{l}186 \\
182 \\
186\end{array}$ & $\begin{array}{l}141.7 \\
149.4 \\
145.0\end{array}$ & $\begin{array}{l}21.5 \\
17.5 \\
20.1\end{array}$ & 0.1967 \\
\hline TAD (mmHg) & $\begin{array}{c}\mathrm{F} \\
\mathrm{M} \\
\text { Total }\end{array}$ & $\begin{array}{l}27 \\
20 \\
47\end{array}$ & $\begin{array}{l}50 \\
67 \\
50\end{array}$ & $\begin{array}{l}99 \\
97 \\
99\end{array}$ & $\begin{array}{l}75.2 \\
78.6 \\
76.7\end{array}$ & $\begin{array}{c}14.7 \\
8.8 \\
12.5\end{array}$ & 0.3667 \\
\hline
\end{tabular}




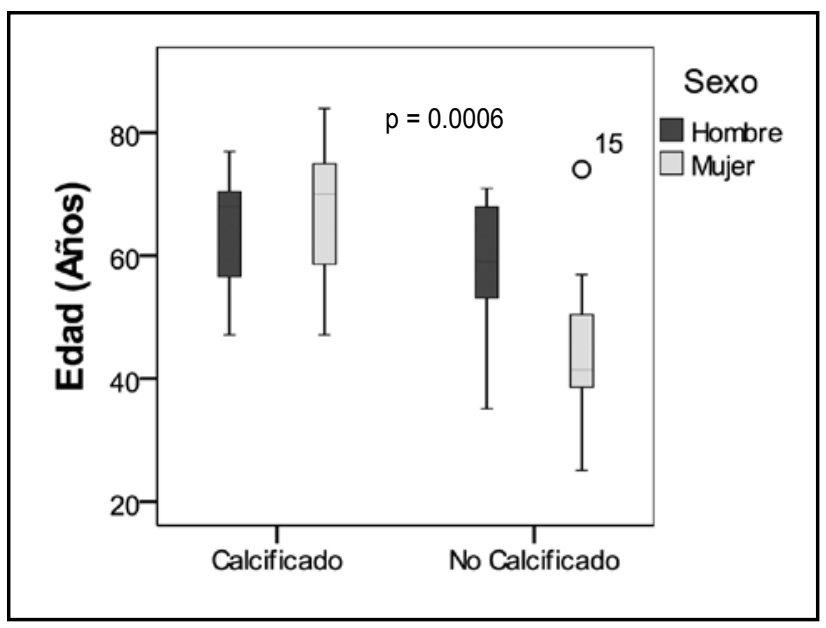

Figura 2. Boxplot de la edad de los pacientes por sexo según grupo de calcificación.

tes mellitus, 98\% (n:46) tenían diagnóstico de hipertensión arterial, $11 \%$ (n:5) referían antecedente de enfermedad coronaria y sólo 4\% (n:2) habían presentado un accidente cerebrovascular. El antecedente de tabaquismo estuvo presente en $38 \%$ (n:18).

Los medicamentos que más consumían los pacientes fueron diuréticos $(51 \%)$, calcioantagonistas $(50 \%)$, calcio $(36 \%)$, betabloqueadores $(28 \%)$, antagonista de receptores de angiotensina II (31.9\%), inhibidores de la enzima convertidora de angiotensina (IECA) (23\%) y estatinas (23\%). La Tabla 3 muestra los resultados de laboratorio por sexo, realizados a los pacientes. Se encontró una diferencia estadísticamente significativa entre los valores de colesterol

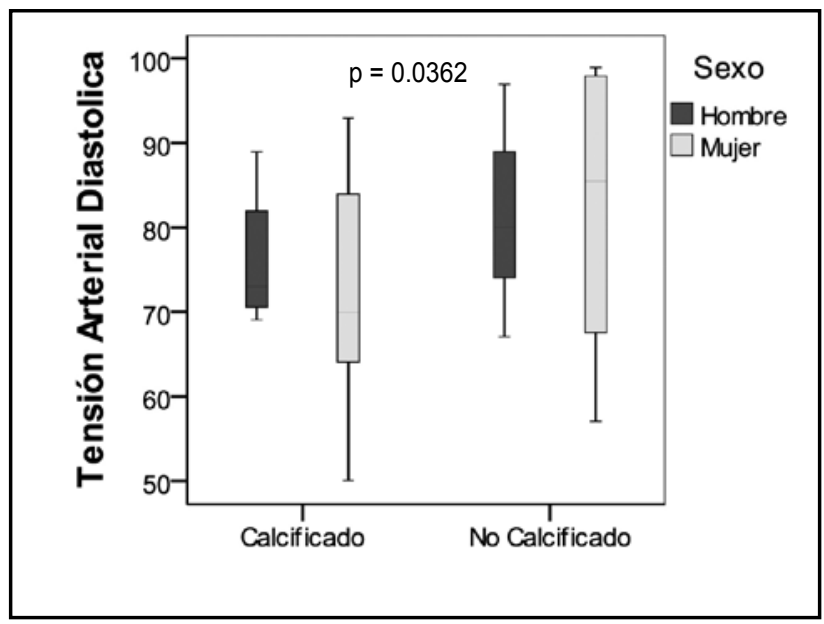

Figura 3. Boxplot de la tensión arterial diastólica $(\mathrm{mmHg})$ de los pacientes por sexo, según grupo de calcificación.

total y colesterol HDL, entre hombres y mujeres $(\mathrm{p}<0.05)$.

En las radiografías lumbares laterales se encontró que $63.8 \%$ de los pacientes presentaban algún segmento calcificado. Del total de pacientes que no presentaba ningún segmento calcificado, $52.9 \%$ eran hombres y $47.1 \%$ eran mujeres. Los segmentos más calcificados en mujeres fueron L1 y L2 y en hombres fueron L4 y L3 (Figura 4).

$\mathrm{Al}$ realizar el puntaje compuesto de CAA, se observa que las mujeres presentaron un puntaje mayor en comparación con los hombres. Estas diferencias se evidencian a partir del cuartil 2 y 3 (Figura 5). El puntaje compuesto promedio de CAA fue de 6.04 para toda la población.

En una segunda etapa se realizó un análisis de regresión

Tabla 3. Datos de laboratorio de los pacientes en hemodiálisis.

\begin{tabular}{|c|c|c|c|c|c|c|c|}
\hline Variables & Sexo & $\mathbf{N}$ & Mínimo & Máximo & Promedio & $\pm \mathrm{DS}$ & $\mathbf{P}$ \\
\hline Calcio (mg/dL) & $\begin{array}{c}\mathrm{F} \\
\mathrm{M} \\
\text { Total }\end{array}$ & $\begin{array}{l}27 \\
20 \\
47 \\
\end{array}$ & 7.8 & 11.0 & $\begin{array}{l}9.1 \\
8.7 \\
8.9\end{array}$ & $\begin{array}{l}0.8 \\
0.6 \\
0.7\end{array}$ & 0.0546 \\
\hline PTH (pg/ml) & $\begin{array}{c}\mathrm{F} \\
\mathrm{M} \\
\text { Total }\end{array}$ & $\begin{array}{l}27 \\
20 \\
47\end{array}$ & 19.8 & 854.6 & $\begin{array}{l}221.5 \\
394.5 \\
295.1\end{array}$ & $\begin{array}{l}181.5 \\
429.7 \\
319.9\end{array}$ & 0.0661 \\
\hline Fosforo $(\mathrm{mg} / \mathrm{dL})$ & $\begin{array}{c}\mathrm{F} \\
\mathrm{M} \\
\text { Total }\end{array}$ & $\begin{array}{l}27 \\
20 \\
47\end{array}$ & 2.0 & 8.8 & $\begin{array}{l}4.8 \\
4.3 \\
4.6\end{array}$ & $\begin{array}{l}1.7 \\
1.1 \\
1.5\end{array}$ & 0.2537 \\
\hline Colesterol total $(\mathrm{mg} / \mathrm{dL})$ & $\begin{array}{c}\mathrm{F} \\
\mathrm{M} \\
\text { Total }\end{array}$ & $\begin{array}{l}27 \\
20 \\
47\end{array}$ & 137.0 & 244.0 & $\begin{array}{l}191.5 \\
171.5 \\
183.0\end{array}$ & $\begin{array}{l}28.9 \\
36.5 \\
33.5\end{array}$ & 0.0413 \\
\hline C-HDL (mg/dL) & $\begin{array}{c}\mathrm{F} \\
\mathrm{M} \\
\text { Total }\end{array}$ & $\begin{array}{l}17 \\
16 \\
33\end{array}$ & 22.0 & 132.0 & $\begin{array}{l}68.8 \\
44.0 \\
56.8\end{array}$ & $\begin{array}{l}28.4 \\
15.8 \\
26.0\end{array}$ & $0.0044^{*}$ \\
\hline C-LDL(mg/dL) & $\begin{array}{c}\mathrm{F} \\
\mathrm{M} \\
\text { Total }\end{array}$ & $\begin{array}{l}17 \\
16 \\
33\end{array}$ & 58.0 & 148.0 & $\begin{array}{l}98.8 \\
93.0 \\
96.0\end{array}$ & $\begin{array}{l}28.3 \\
30.5 \\
29.1\end{array}$ & 0.5773 \\
\hline Triglicéridos (mg/dL) & $\begin{array}{c}\mathrm{F} \\
\mathrm{M} \\
\text { Total }\end{array}$ & $\begin{array}{l}27 \\
20 \\
47\end{array}$ & 40.0 & 626.0 & $\begin{array}{l}160.2 \\
174.8 \\
166.4\end{array}$ & $\begin{array}{c}116.0 \\
81.4 \\
101.9\end{array}$ & 0.6333 \\
\hline Calcio $\mathrm{x}$ fósforo & $\begin{array}{c}\mathrm{F} \\
\mathrm{M} \\
\text { Total }\end{array}$ & $\begin{array}{l}27 \\
20 \\
47\end{array}$ & 18.2 & 77.4 & $\begin{array}{l}43.9 \\
37.8 \\
41.3\end{array}$ & $\begin{array}{l}15.4 \\
10.9 \\
13.9\end{array}$ & 0.1419 \\
\hline
\end{tabular}




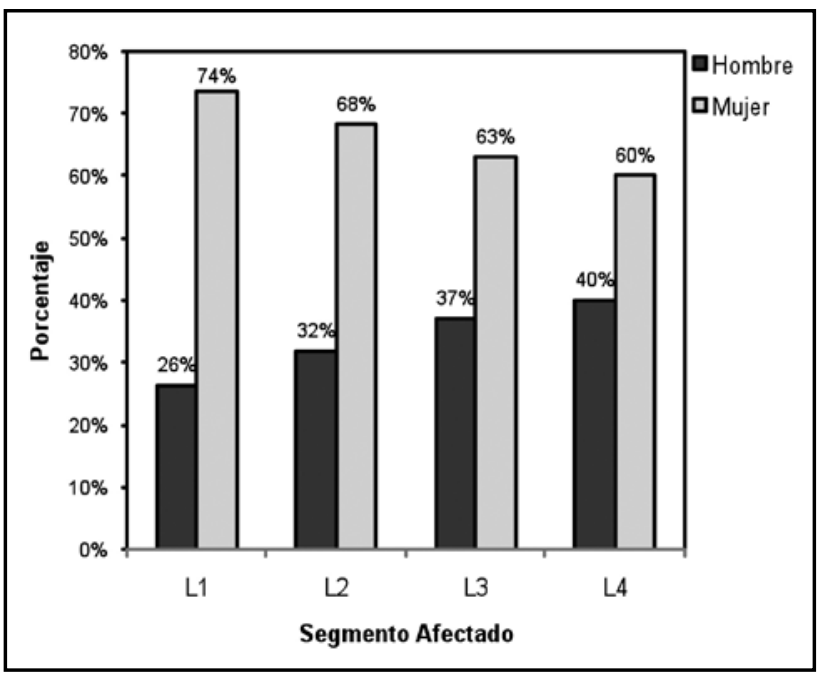

Figura 4. Segmentos afectados en radiografía lumbar lateral.

Tabla 4. Coeficientes estimados para un modelo de regresión logístico múltiple.

\begin{tabular}{|l|c|c|c|c|}
\hline Variable & Coeficiente & $\begin{array}{c}\text { Error } \\
\text { estándar }\end{array}$ & Wald & P \\
\hline Edad & 0.143 & 0.053 & 2.674 & $0.00748^{*}$ \\
Sexo masculino & -1.412 & 1.157 & -1.221 & 0.22219 \\
Tensión arterial diastólica & -0.151 & 0.063 & -2.379 & $0.01738^{*}$ \\
Uso de IECA & -1.731 & 1.233 & -1.404 & 0.16031 \\
Historia de diabetes mellitus & 2.715 & 1.428 & 1.901 & 0.05726. \\
Antecedente de tabaquismo & 1.466 & 1.101 & 1.331 & 0.18311 \\
\hline
\end{tabular}

logística binomial. De acuerdo con las medidas de bondad de ajuste, se encontró que el modelo representado por las variables edad, sexo, tensión arterial diastólica baja, consumo de IECA, antecedentes de diabetes y tabaquismo contiene los factores de riesgo que mayormente explican la prevalencia de calcificación de aorta abdominal, cuyas estimaciones se presentan en la Tabla 4.

Durante el estudio falleció una paciente que presentaba el mayor puntaje de calcificación de la aorta abdominal.

\section{Discusión}

Varios factores de riesgo tradicionales y no tradicionales se han relacionado con la progresión de calcificación vascular en pacientes en hemodiálisis, el cual es un predictor de morbimortalidad cardiovascular; dentro de los cuales se han implicado la edad, la hiperfosfatemia, el incremento del producto de calcio y fósforo, la hipercalcemia, el hiperparatiroidismo, la diabetes mellitus, el exceso de calcio transferido en diálisis y la duración de la diálisis $(7,9,10$, $12,14,19)$. En el modelo de regresión logística realizado en el estudio se encontró que a medida que la edad aumenta, la TAD disminuye, si hay antecedente de tabaquismo y diabetes la probabilidad de riesgo de calcificación es mayor. $\mathrm{Si}$ el paciente es hombre y consume IECA, disminuye la

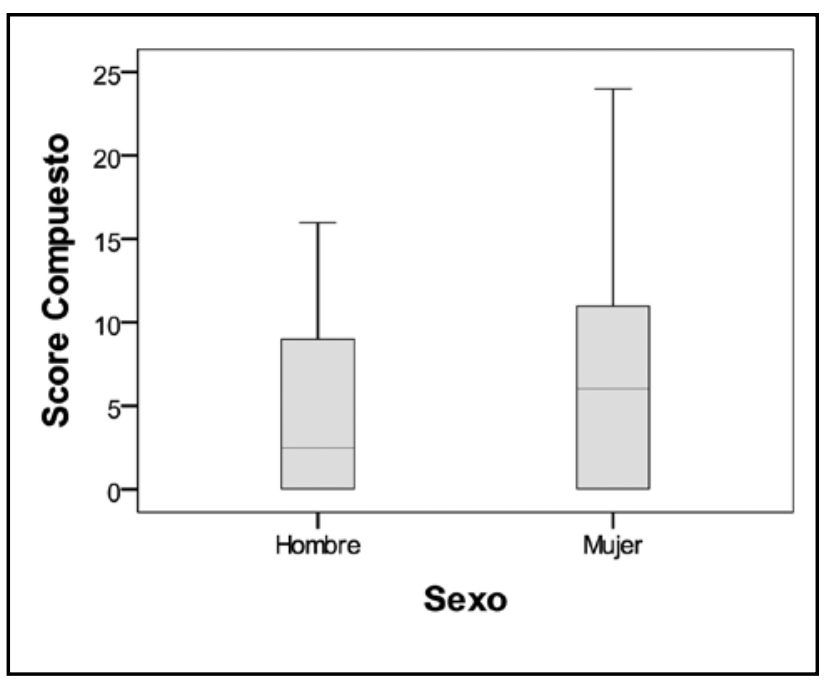

Figura 5. Boxplot del puntaje compuesto de calcificación de aorta abdominal en radiografía lumbar.

probabilidad de riesgo de calcificación.

El uso de de tomografía para medición de calcificaciones aórticas es un método sensible y provee mediciones de calcificación cuantitativa; sin embargo, es un instrumento costoso y con una exposición mayor de radiación. Algunos trabajos han evaluado la radiografía para detección de calcificaciones, lo cual puede ser un estudio inicial y puede potencialmente distinguir entre calcificación íntima y media $(4,28)$. En el estudio publicado por Kauppila y col. (8) en el seguimiento de calcificación aórtica abdominal en la cohorte de Framingham, la prevalencia de calcificaciones aorticas en hombres fue estimada en $37 \%$ y en mujeres de $27 \%$ con radiografía lumbar lateral; sin embargo, en el seguimiento a 25 años se evidenció que ese porcentaje se incrementaba a $86 \%$ en ambos géneros. En este mismo estudio se propone el puntaje para CAA, evaluando el segmento afectado (L1 a L4), pared afectada (anterior y/o posterior) y el puntaje compuesto (rango:0-24), el cual es utilizado en el estudio CORD y en el presente estudio (10).

Bellasi y col (29) evaluaron el puntaje compuesto de CAA en 140 paciente en hemodiálisis con un promedio de edad de 55 años y tiempo de hemodiálisis de 2.7 años. El promedio del puntaje fue de 4.4. Toussaint y col, (27) encontraron en 132 pacientes un score de 11. Nuestro estudio reporta un score promedio de calcificación de aorta abdominal menor (6.04), explicado posiblemente, porque el promedio de edad de los pacientes y el tiempo de diálisis fue menor.

Autopsias realizadas en pacientes con enfermedad renal crónica en hemodiálisis, describen calcificaciones en varios tejidos, incluyendo calcificación vascular en 50-80\% de estos pacientes (11). Otros estudios reportan que entre 40-95\% de los pacientes tienen calcificaciones aórticas dependiendo de la edad, presencia de comorbilidades y métodos de detección de las calcificaciones vasculares (7, 
$10,20-27)$. Este estudio reporta que $63.8 \%$ de los pacientes en hemodiálisis tienen presencia de calcificaciones de aorta abdominal detectada por radiografía lumbar lateral.

Se encontró que a medida que la edad aumenta la probabilidad de riesgo de CAA se incrementa; sin embargo, no hubo asociación con el tiempo en hemodiálisis, valores de PTH, fósforo, calcio, colesterol, triglicéridos, producto calcio $\mathrm{x}$ fósforo y enfermedades asociadas como hipertensión arterial, enfermedad coronaria y accidente cerebrovascular. Este resultado podría ser explicado por el pequeño número de pacientes del estudio; sin embargo, esta relación en diferentes estudios ha sido inconsistente. Algunos estudios han reportado una correlación entre el fósforo, PTH, calcio y calcificación vascular $(7,20,30,31)$; sin embargo, otros no han reportado estas asociaciones y las razones de estas inconsistencias son poco claras $(10,17,27)$.

Se determinó una disminución significativa de la TAD de los pacientes con CAA (P: 0.036). Este resultado fue reportado por Guerin y col (13) donde mostraron un incremento de la presión sistólica en los pacientes con calcificaciones vasculares y paralelamente una disminución en TAD con incremento por lo tanto de la presión de pulso, asociado a un incremento de la velocidad de la onda de pulso aórtica de $9 \mathrm{~m} / \mathrm{seg}$ en el grupo con menor calcificación a $13 \mathrm{~m} / \mathrm{seg}$ en el grupo más calcificado. El incremento de la presión de pulso indirectamente sugiere disminución de la distensibilidad de la pared de la aorta, lo cual podría estar en relación a calcificación de su pared.

Las recomendaciones para determinar calcificaciones vasculares en pacientes en hemodiálisis como parte de la práctica clínica es aún incierta, debido a que no hay protocolos o algoritmos basados en la evidencia para las estrategias terapéuticas en caso de detectar CAA. Sin embargo, la detección de CAA puede ser útil para mejorar la identificación de los pacientes en diálisis, quienes se benefician de tratamientos más agresivos de factores de riesgo (control estricto de alteraciones del metabolismo, evitar la ingesta exógena de calcio excesiva, disminuir aporte de calcio en el líquido dializado, control glicémico y de dislipidemia) (27).

Los autores somos conscientes de las limitaciones del estudio debido al número de pacientes y que fue realizado en una sola unidad renal. Igualmente, la radiografía lumbar no es el estudio imagenológico con mayor sensibilidad y especificidad para detectar calcificaciones vasculares. Sin embargo, este estudio provee datos muy importantes sobre la prevalencia de CAA en pacientes sometidos a hemodiálisis y variables asociadas en estos pacientes.

En conclusión, la calcificación de aorta abdominal se presenta en un porcentaje alto de pacientes con ERC en hemodiálisis. A mayor edad y menor TAD el riesgo de prevalencia de calcificación es mayor. La radiografía lumbar como lo menciona las guías K/DIGO publicadas, puede ser un instrumento de costo menor para ser tenida en cuenta en los pacientes en hemodiálisis como herramienta para el diagnóstico de calcificaciones y complicaciones y/o riesgo cardiovascular.

\section{Agradecimientos}

Unidad Renal -Fresenius Medical Care- Centro Médico de Imbanaco y al profesor Reynaldo Carvajal por sus asesoría estadística.

Dra. Claudia Birchenall, epidemióloga de Fresenius Medical Care por sus valiosos aportes.

Fresenius Medical Care, por la colaboración para el presente estudio.

Ninguno.

\section{Declaración de conflicto de intereses}

\section{Referencias}

1. Calvanese Nicotina. Estilos de afrontamiento y adaptación al tratamiento en pacientes sometidos a hemodiálisis. Nefrología latinoamericana 2003; 1.

2. Goodman WG, London G. On Behalf of the Vascular Calcification Work Group. Vascular calcification in chronic kidney disease. Am JKidney Dis 2004; 43: 572-579

3. Blacher J, Guerin AP, Pannier B, Marchais S, London G. Arterial calcifications, arterial stiffness, and cardiovascular risk in end-stage renal disease. Hypertension 2001; 38: 938-942.

4. London GM, Guerin AP, Marchais SJ, Metivier F, Pannier B, Adda H. Arterial media calcification in end-stage renal disease: impact on all-cause and cardiovascular mortality. Nephrol Dial Transplant 2003; 18: 1731-1740.

5. Chertow GM, Burke SK, Raggi P. Treat to Goal Working Group. Sevelamer attenuates the progression of coronary and aortic calcification in hemodialysis patients. Kidney Int 2002; 62: 245-252.

6. Asmus HG, Braun J, Krause R, Brunkhorst R, Holzer H, Schulz W, Neumayer HH, Raggi P, Bommer J. Two year comparison of sevelamer and calcium carbonate effects on cardiovascular calcification and bone density. Nephrol Dial Transplant 2005; 20: 1653-1661.

7. Yamada K, Fujimoto S, Nishiura R. Risk factors of the progression of abdominal aortic calcification in patients on chronic haemodialysis, Nephrol Dial Transplant 2007; 22: 2032-2037.

8. Kauppila LI, Polak JF, Cupples LA, Hannan MT, Kiel DP, Wilson PW. New índices to classify location, severity and progression of calcific lesions in the abdominal aorta: a 25 years follow-up study. Atherosclerosis 1997; 132: 245-250.

9. Wilson PW, Kauppila L, O’Donnel CJ, Kiel D, Hannan M,Polak JM, et al Abdominal aortic calcific deposits are an important predictor of vascular morbidity and mortality. Circulation 2001; 103: 1529-1534.

10. Honkanen E, Kauppila L, Wikstrom B, Rensma P,Krzesinski JM, Aasarod $\mathbf{K}$, et al. Abdominal aortic calcification in dialysis patients: results of the CORD study. Nephrol Dial Transplant 2008; 23: 4009-4015.

11. Kuzela DC, Huffer WE, Conger JD, Winter SD, Hammond WS. Soft-tissue calcification in chronic dialysis patients. Am J Pathol 1977; 86: 403-424.

12. Taniwaki H., Ishimura E., Tabata T.Aortic calcification in haemodialysis patients with diabetes mellitus. Nephrol Dial Transplant 2005; 20: 2472-2478.

13. Guerin AP, London GM., Marchars SJ, Metivier F. Arterial Stiffenning and vascular calcifications in end-stage renal disease. Nephrol Dial Transplant 2000; 15: $1014-1021$.

14. Moe SM, Drüeke TB, Block GA, et al. KDIGO clinical practice guideline for the diagnosis, evaluation, prevention, and treatment of Chronic Kidney DiseaseMineral and Bone Disorder (CKD-MBD). Kidney Disease: Improving Global Outcomes (KDIGO). CKD-MBD Work Group Kidney Int 2009; 113: 1-130

15. Walsh CR, Cupples LA, Levy D, et al. Abdominal aortic calcific deposits are associated with increased risk for congestive heart failure: The Framingham Heart Study. Am Heart J 2002; 144: 733-739.

16. Matsuoka M, Iseki K, Tamashiro M, et al. Impact of high coronary artery calcification score (CACS) on survival in patients on chronic hemodialysis. Clin Exp Nephrol 2004; 8: 54-58.

17. Moe S, O’Neill K, Fineberg N, Persohn S, Ahmed S, Garrett P, Meyer C. Assessment of vascular calcification in ESRD patients using spiral CT. Nephrol Dial Transplant 2003; 18: 1152-1158.

18. Adragao T, Pires A, Lucas C, et al. A simple vascular calcification score predicts cardiovascular risk in haemodialysis patients. Nephrol Dial Transplant 2004: 19; 1480- 1488,

19. Nieto I, Mahecha P. Asociación entre hiperfosfatemia y mortalidad en los pacientes con insuficiencia renal crónica terminal en terapia de reemplazo de la función renal con diálisis peritoneal o hemodiálisis. Un estudio de la Unidad Renal de Villavicencio 1999-2003. Acta Med Colomb 2005; 30: 14-18. 
20. Shigematsu T, Kono T, Satoh K et al. Phosphate overload accelerates vascular calcium deposition in end-stage renal disease patients. Nephrol Dial Transplant 2003; 18: 86-9.

21. Okuno S, Ishimura E, Kitakani K et al. Presence of abdominal aortic calcification is significantly associated with all-cause and cardiovascular mortality in maintenance hemodialysis patients. Am J Kidney Dis 2007; 49: 417-25.

22. Toussaint ND, Lau KK, Strauss BJ, Polkinghorne KR, Kerr PG. Relationship between vascular calcification, arterial stiffness and bone mineral density in a cross-sectional study of prevalent Australian haemodialysis patients. Nephrology 2009; 14: 105-12.

23. Ogawa T, Ishida $\mathbf{H}$, Matsuda $\mathbf{N}$ et al. Simple evaluation of aortic arch calcification by chest radiography in hemodialysis patients. Hemodial Int 2009; 13: 301-6.

24. Ogawa T, Ishida H, Akamatsu M et al. Progression of aortic arch calcification and all-cause and cardiovascular mortality in chronic hemodialysis patients. Int Urol Nephro. 2010; 42: 187-94.

25. Fujiu A, Ogawa T, Matsuda N, Ando Y, Nitta K. Aortic arch calcification and arterial stiffness are independent factors for diastolic left ventricular dysfunction in chronic hemodialysis patients. Circulation 2008; 72: 1768-72.
26. London GM, Marchais SJ, Guérin AP, Boutouyrie P, Métivier F, de Vernejoul MC. Association of bone activity, calcium load, aortic stiffness, and calcifications in ESRD. J Am Soc Nephrol 2008; 19:1827-35.

27. Toussaint N, Pedagogos E, Lau K, Heinze S, Becker GJ, Beavis J, Polkinghorne KR, Damasiewicz MJ and Kerr PG. Lateral lumbar X-ray assessment of abdominal aortic calcification in Australian haemodialysis patients Nephrology 2011; 16: 389-395.

28. Goldsmith DJ, Covic A, Sambrook PA et al. Vascular calcification in long-term haemodialysis patients in a single unit: A retrospective analysis. Nephron 1997; 77: 37-43.

29. Bellasi A, Ferramosca E, Muntner P et al. Correlation of simple imaging test and coronary artery calcium measured by computed tomography in hemodialysis patients. Kidney Int 2006; 70: 1623-8.

30. Goodman WG, Goldin J, Kuizon BD et al. Coronary-artery calcification in young adults with end-stage renal disease who are undergoing dialysis. $N$ Engl $J$ Med 2000; 342: 1478-83.

31. Raggi P, Boulay A, Chasan-Taber $\mathbf{S}$ et al. Cardiac calcification in adult hemodialysis patients. A link between end-stage renal disease and cardiovascular disease? J Am Coll Cardiol 2002; 39: 695-701. 